Proc. of 12th International Workshop on Positron and Positronium Chemistry, August 28-September 1, 2017, Lublin, Poland

\title{
Positronium Formation in Nanostructured Metals
}

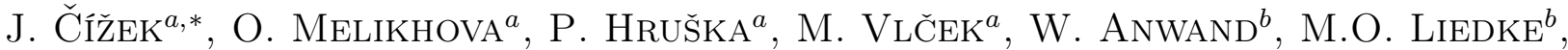
M. NOVOTNÝ ${ }^{c}$ AND J. BULÍr $\check{R}^{c}$

${ }^{a}$ Faculty of Mathematics and Physics, Charles University, V Holešovičkách 2, 18000, Praha 8, Czech Republic

${ }^{b}$ Institute of Radiation Physics, Helmholtz-Zentrum Dresden-Rossendorf,

Bautzner Landstr. 400, 01328 Dresden, Germany

${ }^{c}$ Institute of Physics, Academy of Sciences of the Czech Republic, Na Slovance 2, 18221 Prague 8, Czech Republic

Positronium formation in $\mathrm{Au}$ films was studied using a magnetically guided continuous slow positron beam with variable energy. Black Au film with porous morphology was compared with conventional smooth Au film. In the smooth Au film positronium is formed on the film surface only. The black Au film exhibits porous sub-surface region containing micro-cavities interconnected with surface. Positronium is formed on inner surfaces of microcavities in the sub-surface region and travels through interconnected cavities towards the surface. $3-\gamma$ annihilation of ortho-positronium leaves a clear signature in two-dimensional coincidence Doppler broadening spectra. Measures of $3-\gamma$ annihilation contribution calculated from single $\gamma$-ray and coincidence Doppler broadening spectra were calculated and compared.

DOI: 10.12693/APhysPolA.132.1579

PACS/topics: 78.70.Bj, 41.75.Fr, 36.10.Dr

\section{Introduction}

Thermal evaporation of metals in a low pressure $(\approx$ $100 \mathrm{~Pa}$ ) of a non-interacting gas enables to prepare porous films known as black metals [1]. Light incident on a black metal film is absorbed in multiple reflections in fractal-like structure of percolated micro-cavities with various sizes. This makes black metals highly absorbing light in the visible to infrared spectral region [2]. Light absorbers are important in optical applications for preventing crosstalk between optical connections. Nevertheless a full omnidirectional absorption of incident light in the visible to the infrared spectral region has not been achieved yet [3]. The morphology and size distribution of micro-cavities are crucial for performance of black metals absorbers [2]. Positronium (Ps) is an excellent probe for non-destructive characterization of micro-cavities in porous materials and enables precise determination of their size distribution [4]. Inside conventional metals Ps does not form since any bound state of positron and electron is quickly destroyed by the screening of conduction electrons [5]. However, a thermalized positron escaping from a metal through inner surface into a cavity may form Ps by picking an electron on the surface [6]. This process was examined in the present work by single $\gamma$-ray and coincidence Doppler broadening (CDB) spectroscopy [7] carried out on a variable energy slow positron beam. Black $\mathrm{Au}$ film prepared by thermal evaporation in $\mathrm{N}_{2}$ atmosphere was compared with smooth film deposited in vacuum.

\section{Experimental details}

Conventional smooth $\mathrm{Au}$ film with thickness of $\approx 900 \mathrm{~nm}$ was prepared by thermal deposition on glass

*corresponding author; e-mail: Jakub.cizek@mff.cuni.cz substrates in vacuum. Black Au film with the same thickness was prepared by thermal deposition on glass substrate in $\mathrm{N}_{2}$ atmosphere at the pressure of $200 \mathrm{~Pa}$.

Morphology of films was characterized by scanning electron microscope (SEM) Philips XL 30 CP equipped with energy dispersive X-ray spectrometer (EDX).

Positron annihilation studies were performed on a magnetically guided slow positron beam with energy of incident positrons variable in the range from $30 \mathrm{eV}$ to $35 \mathrm{keV}$ [8]. The Doppler broadening of annihilation photo-peak was measured either by a single high purity Ge (HPGe) detector with energy resolution of $1.09 \mathrm{keV}$ at $511 \mathrm{keV}$ or by two HPGe detectors in coincidence using a digital CDB spectrometer described in Ref. [9]. The digital CDB spectrometer exhibits overall energy resolution of $0.99 \mathrm{keV}$ at the annihilation photo-peak. Pulses from HPGe detectors are sharpened by a spectroscopy amplifier in order to improve their signal-to-noise ratio and sampled by a couple of 12-bit digitizers Acqiris DC440 (Agilent Technology) with the sampling rate of $420 \mathrm{MHz}$. Analysis of sampled waveforms is performed off-line by dedicated software $[9,10]$. Measurement in coincidence enables to reduce background by three orders of magnitude [11]. Moreover, digital shape filters which are applied during analysis of sampled waveforms reject pulses with shape distorted by pile-up effects or ballistic deficit during charge collection in the detector [9]. As a consequence spectra with a high clarity and very low background were obtained using the digital CDB spectrometer [12].

\section{Results and discussion}

Film deposited in vacuum exhibits smooth mirror-like surface with a high reflectivity. As an example Fig. 1a shows an image of smooth Au film deposited in vacuum. On the other hand, black Au film shown in Fig. 1b exhibits porous structure and its surface appears dark since 
incident light is absorbed in multiple reflections in interconnected micro-cavities. Figure 1c shows a view on the same black $\mathrm{Au}$ film but at the back side, i.e. through the glass substrate when the film is turned over. Obviously when looking through the glass substrate the film appears as a smooth mirror-like layer with a high reflectivity. It indicates that on the substrate the film grows as a smooth layer which becomes more and more porous with increasing thickness.

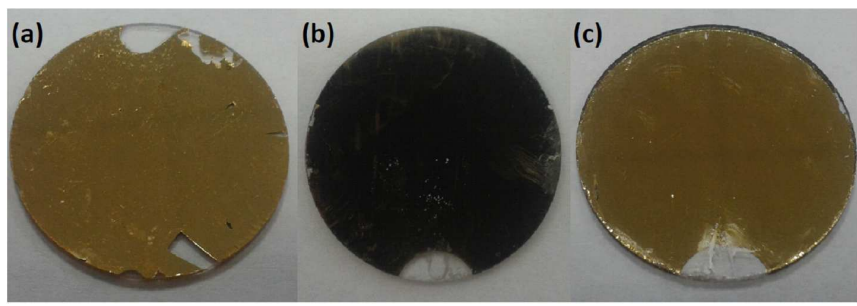

Fig. 1. Appearance of Au films: (a) smooth Au film, (b) black Au film - front side, (c) black Au film back side (look through the glass substrate).

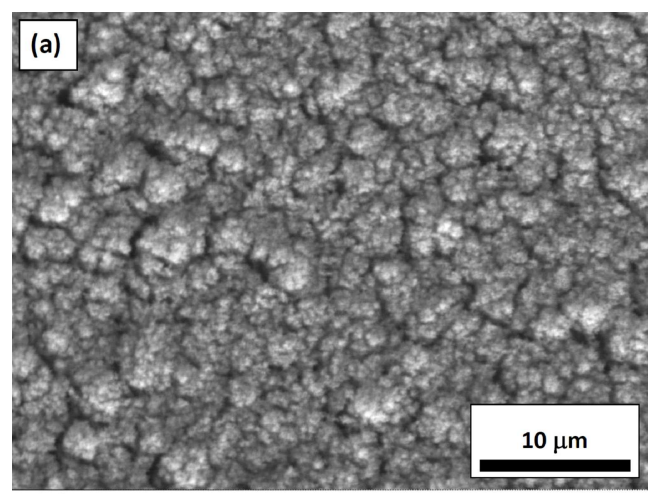

(b)

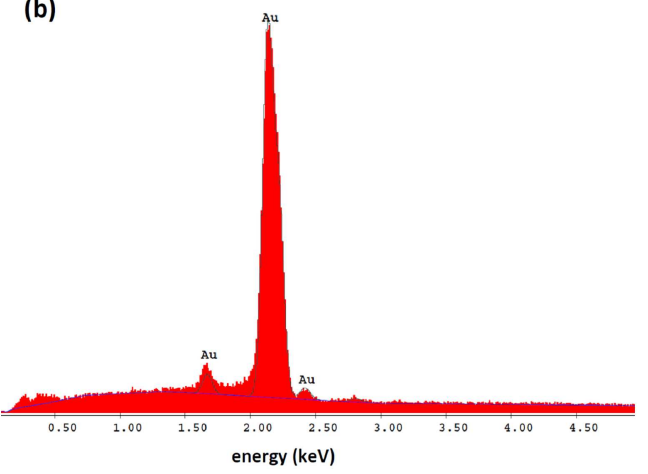

Fig. 2. Black Au film: (a) SEM micrograph of surface, (b) EDX spectrum.

Figure 2a shows a SEM image of the surface of black $\mathrm{Au}$ film. It is clear that the film exhibits porous structure with a wide size distribution of micro-cavities. EDX spectrum for the black Au film plotted in Fig. 2b shows no contribution from any chemical element but $\mathrm{Au}$. It confirms that the black $\mathrm{Au}$ film is pure gold and does

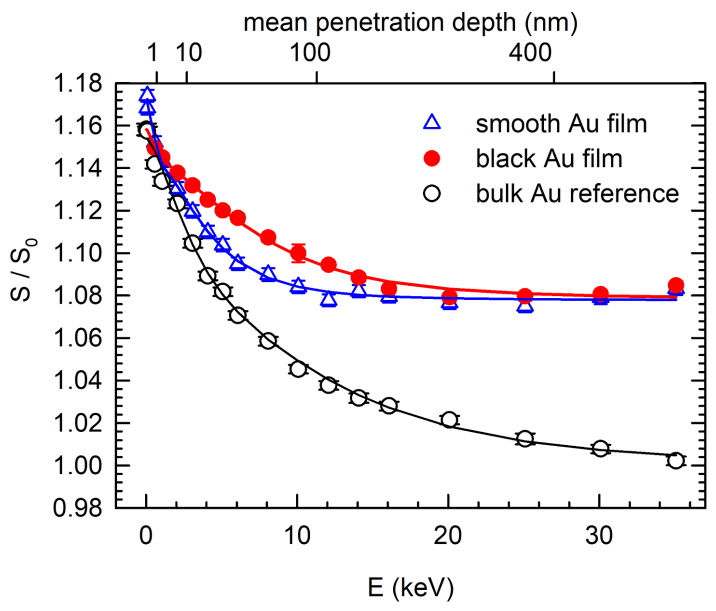

Fig. 3. Dependence of the $S$ parameter on the energy $E$ of incident positrons for smooth and black Au films. The $S(E)$ curve for a well annealed bulk Au reference is plotted in the figure as well. Solid lines are model curves calculated by VEPFIT.

not contain any oxide or nitride layer on the surface.

The dependence of the $S$ parameter on the energy $E$ of incident positrons for the smooth and black $\mathrm{Au}$ film is plotted in Fig. 3. The $S(E)$ curve for a well annealed $\left(100{ }^{\circ} \mathrm{C} / 1 \mathrm{~h}\right)$ bulk $\mathrm{Au}$ reference [13] is plotted in the figure as well. The $S$ parameter values were always normalized to the bulk parameter value $S_{0}$ for the well annealed $\mathrm{Au}$ reference. At low positron energies almost all positrons are annihilated at the surface and the $S$ parameter, therefore, corresponds to the surface positron state. With increasing energy positrons penetrate deeper and deeper into the film and the fraction of positrons diffusing back to the surface decreases. It is reflected by a decrease of $S$ from the surface value to the bulk value corresponding to the situation when all positrons are annihilated inside the film and the fraction of positrons diffusing back to the surface is negligible.

Fitting of the $S(E)$ curve for the Au reference by the VEPFIT code [14] revealed positron diffusion length of $134 \pm 1 \mathrm{~nm}$. This value falls into the range $100-150 \mathrm{~nm}$ expected for positron diffusion in defect free metals [15]. It confirms that the reference $\mathrm{Au}$ sample exhibits very low concentration of defects and majority of positrons is annihilated in the free state.

Both smooth and black $\mathrm{Au}$ films contain open volume defects as demonstrated by higher $S$ parameter values and shortened positron diffusion length. The smooth $\mathrm{Au}$ film can be fitted by VEPFIT [14] assuming a single layer and the mean positron diffusion length $L_{+}=24 \pm 1 \mathrm{~nm}$ was obtained from fitting. The black Au film consists of two layers: (i) "bulk-like" region which is characterized by the $S$ parameter and positron diffusion length similar to those for the smooth Au film and (ii) "sub-surface" region which differs from the smooth film. The thickness of the sub-surface region obtained from fitting is $79 \pm 3 \mathrm{~nm}$ and it is characterized by the positron diffusion length 
$L_{+}=62 \pm 4 \mathrm{~nm}$. The difference between the black and smooth $\mathrm{Au}$ film can be well visualized by relative differences of $S$ parameters $\left(S_{\text {black }}-S_{\text {smooth }}\right) / S_{\text {smooth }}$ plotted in Fig. 4. The black Au film differs from the smooth film by enhanced $S$ parameter in the sub-surface region. Hence, the sub-surface region in the black Au film exhibits higher $S$ parameter compared to the smooth film and at the same time longer positron diffusion length. This can be explained only by contribution of Ps formed on inner surfaces of micro-cavities in the sub-surface region of the black Au film. Narrow momentum distribution of para-positronium (p-Ps) contribution [16] enhances the $S$ parameter while Ps diffusion in cavities interconnected with surface leads to a longer diffusion length determined by VEPFIT.

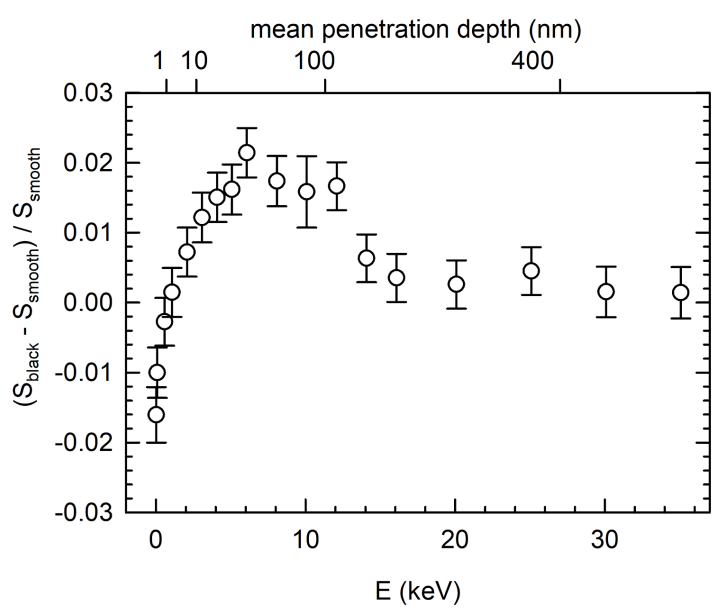

Fig. 4. The relative difference of $S$ parameters between the black and the smooth film $\left(S_{\text {black }}-S_{\text {smooth }}\right) / S_{\text {smooth }}$.

This picture was verified by measurement of the contribution of $3-\gamma$ annihilations to energy spectra of annihilation radiation. Ortho-positronium (o-Ps) in vacuum decays into $3 \gamma$-rays with continuous energy distribution satisfying the relation $E_{1}+E_{2}+E_{3}=2 m_{0} c^{2}$. These $\gamma$-rays contribute to the region below the annihilation photo-peak in the $\gamma$-ray energy spectra. Hence, a measure of $3-\gamma$ contribution can be expressed as "valley-topeak ratio" called also $R$ parameter [17] and defined as:

$$
R=A_{3-\gamma} / A_{2-\gamma},
$$

where $A_{3-\gamma}$ is the area in the region below the annihilation peak and $A_{2-\gamma}$ is the area of the annihilation photo-peak, see Fig. 5a. Here the region for $A_{3-\gamma}$ was selected from 430 to $500 \mathrm{keV}$ and the region for $A_{2-\gamma}$ from 505 to $517 \mathrm{keV}$.

The dependence of the $R$ parameter on the energy of incident positrons is plotted in Fig. 5b. In bulk metals Ps formation is possible on the surface only, since any bound state between positron and electron in bulk is immediately destroyed due to screening by conductive electrons. Hence, the $R$ parameter value for the bulk $\mathrm{Au}$ reference measured at energies of incident positrons $E>10 \mathrm{keV}$ represents a plateau value corresponding to
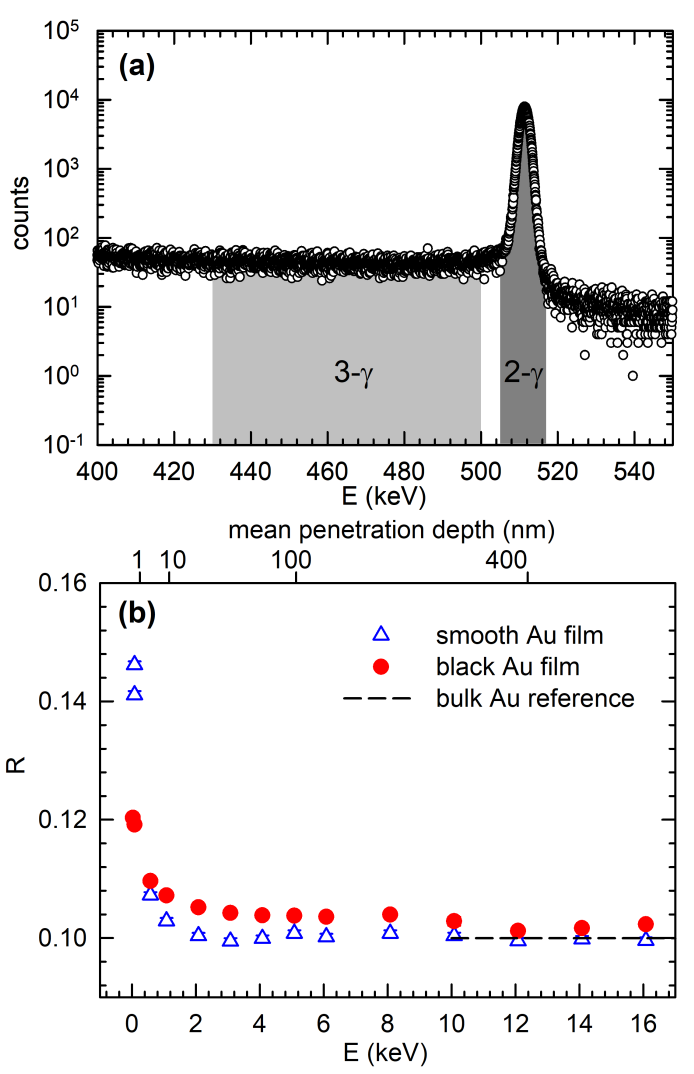

Fig. 5. (a) The regions used for calculation of the $R$ parameter from single $\gamma$-ray spectra, (b) the dependence of the $R$ parameter on the energy of incident positrons. Dashed line indicates the $R$ parameter value for the bulk $\mathrm{Au}$ reference.

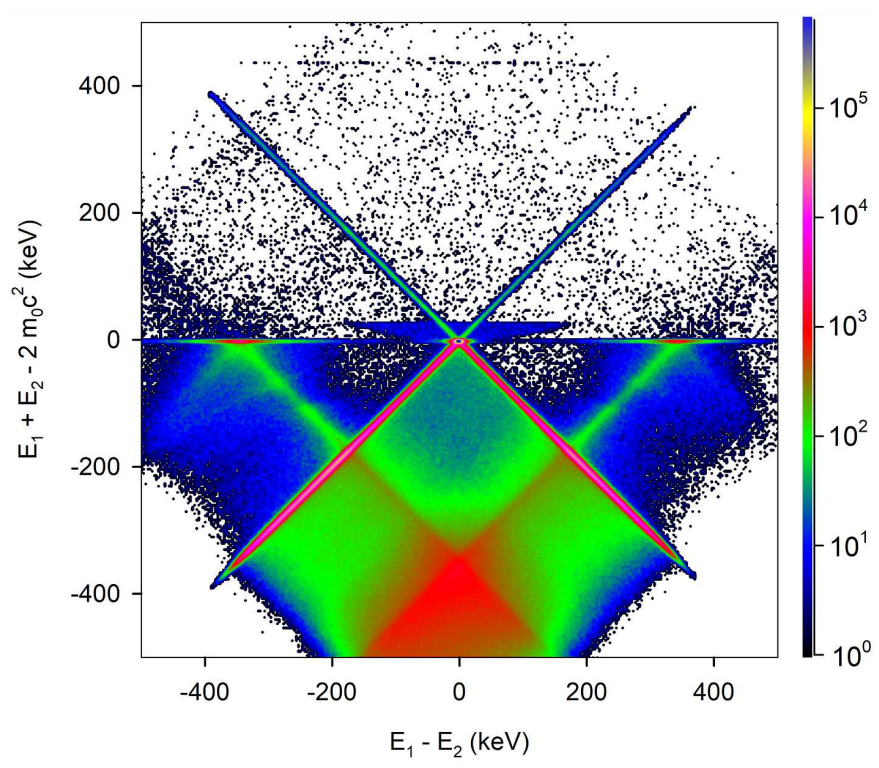

Fig. 6. Two-dimensional CDB spectrum for smooth $\mathrm{Au}$ film measured with energy of incident positrons $E=30 \mathrm{keV}$. 


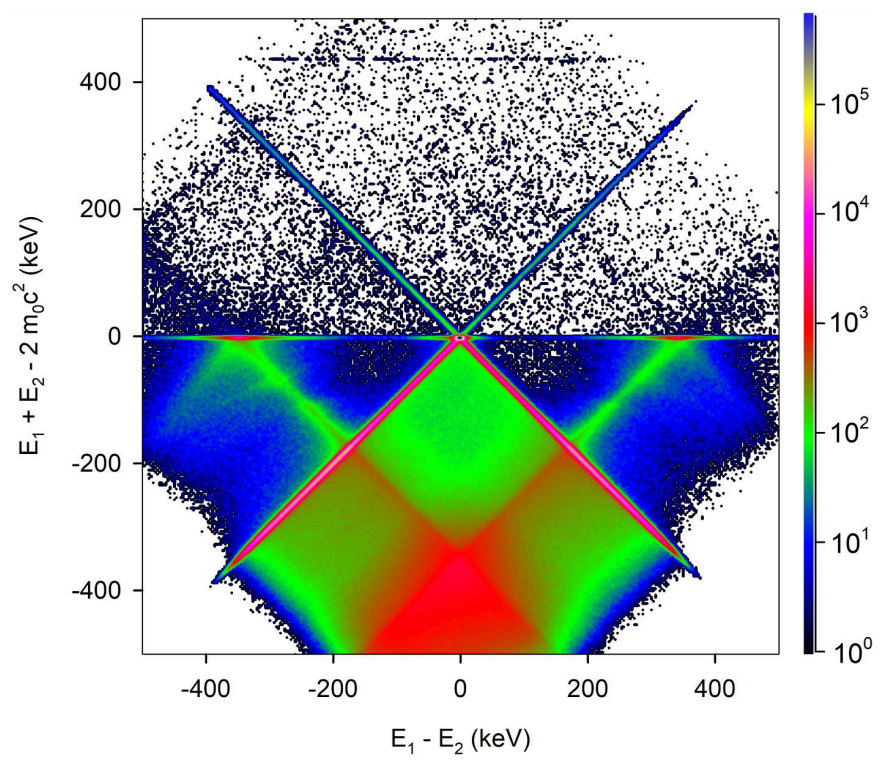

Fig. 7. Two-dimensional CDB spectrum for smooth $\mathrm{Au}$ film measured with energy of incident positrons $E=80 \mathrm{eV}$.

no $3-\gamma$ contribution, see dashed line in Fig. 5b. Nonzero value of $R$ is due to background contributing to the region used for calculation of $A_{3-\gamma}$. The $R(E)$ curve for the smooth $\mathrm{Au}$ film is very similar to that for bulk $\mathrm{Au}$ and sharply decreases to the plateau value measured for the bulk reference. A $3-\gamma$ contribution was observed only at surface of smooth Au film at very low energies of incident positrons, $E<1 \mathrm{keV}$. There are three different mechanisms of Ps formation on metal surface described in literature: (i) emitted Ps: a thermalized positron diffusing back to the surface may pick an electron at the surface forming Ps which is then emitted from the surface to vacuum [6]; (ii) thermally desorbed Ps: a positron trapped in the surface state, i.e. in the potential well at the surface, may escape by coupling with an electron from the surface and forming Ps [18]; (iii) glancing-angle scattering: Ps can be formed also by low energy positrons arriving at the surface from outside at low incident angles. These positrons can be quasi-elastically scattered while picking an electron from the conduction band and forming Ps [19]. All these mechanisms may contribute to the Ps formation on the surface of the smooth $\mathrm{Au}$ film.

From inspection of Fig. 5b it becomes clear that the $R(E)$ curve for the black Au film differs from that for smooth Au film in two respects: (i) the $3-\gamma$ contribution extends to higher energies of incident positrons, i.e. to higher depths. It is obviously due to Ps formed on surfaces of micro-cavities in the porous sub-surface region; (ii) the Ps yield on the surface of black Au film is lower, i.e. $R$ is reduced. The black Au film exhibits higher surface roughness compared to the smooth $\mathrm{Au}$ one. This increases the probability that Ps emitted from certain point on the surface will crash into some hillock on the surface. As a consequence Ps yield on the surface of black $\mathrm{Au}$ is reduced.

Undesired background contributing to the $R$ parameter can be significantly suppressed by measurement in coincidence. Figure 6 shows two-dimensional CDB spectrum for smooth $\mathrm{Au}$ film measured with energy of incident positrons $E=30 \mathrm{keV}$ which ensures that all positrons are annihilated inside the film and the fraction of positrons diffusing back to the surface is negligible. The CDB spectrum in Fig. 6 is plotted in extended energy range which enables to observe signatures of several processes: (i) annihilation photo-peak representing the contribution of $2-\gamma$ annihilation events is located in the center of the spectrum. (ii) Diagonal ridges are caused by $2-\gamma$ events when one annihilation $\gamma$-ray deposited full energy $(511 \mathrm{keV})$ in one detector while the second photon underwent the Compton scattering and escaped from the second detector and by random coincidences with one of the annihilation photon. (iii) Two side peaks located symmetrically around the annihilation photo-peak are caused by $2-\gamma$ events when one annihilation $\gamma$-ray deposited full energy in one detector while the second one interacted in the second detector by the Compton scattering and was scattered back to the first detector. Hence, sum of energies deposited in detectors equals $2 m_{0} c^{2}$ while the energy difference $E_{1}-E_{2}$ is much higher than that caused by electron momentum. The backscattering peaks are centered at position $E_{1}-E_{2}=340 \mathrm{keV}$ corresponding to the scattering angle of $180^{\circ}$. (iv) The horizontal line at $E_{1}+E_{2}-2 m_{0} c^{2}=437 \mathrm{keV}$ is caused by scattering of $1459 \mathrm{keV} \gamma$-ray emitted by long-lived ${ }^{40} \mathrm{~K}$ radioisotope which is always present in the background. Contrary to back-scattering events when the scattering angle is close to $180^{\circ} \mathrm{C}$ the ${ }^{40} \mathrm{~K} \gamma$-ray may arrive from any direction and all scattering angles are therefore possible. This leads to a continuous horizontal line contribution in two-dimensional CDB spectrum. (v) A bowl-like feature above the annihilation photo-peak is a contribution of positron annihilations in flight, i.e. $2-\gamma$ annihilations of non-thermalized positrons [12, 20, 21]. Because of energy conservation $E_{1}+E_{2}-2 m_{0} c^{2}$ cannot be higher than the energy of incident positrons which is $30 \mathrm{keV}$ in the present case. This defines the kinematical cut-off representing "top" of the annihilation-in-flight bowl.

The two-dimensional CDB spectrum measured for the smooth $\mathrm{Au}$ film with low energy of incident positrons $E=80 \mathrm{eV}$ is plotted in Fig. 7. Here majority of positrons was annihilated on the surface. Comparing Figs. 6 and 7 one can notice two important differences between the spectrum for bulk $(E=30 \mathrm{keV})$ and surface $(E=80 \mathrm{eV})$ : (i) the annihilation-in-flight contribution is absent in Fig. 7 since the kinematic cut-off decreases with decreasing energy of incident positrons and the annihilation-inflight contribution diminishes. (ii) The CDB spectrum in Fig. 7 exhibits enhanced contribution below the annihilation photo-peak, i.e. exactly in the region where $3-\gamma$ events contribute. This is caused by $3-\gamma$ annihilation of $o$-Ps formed on the surface. 


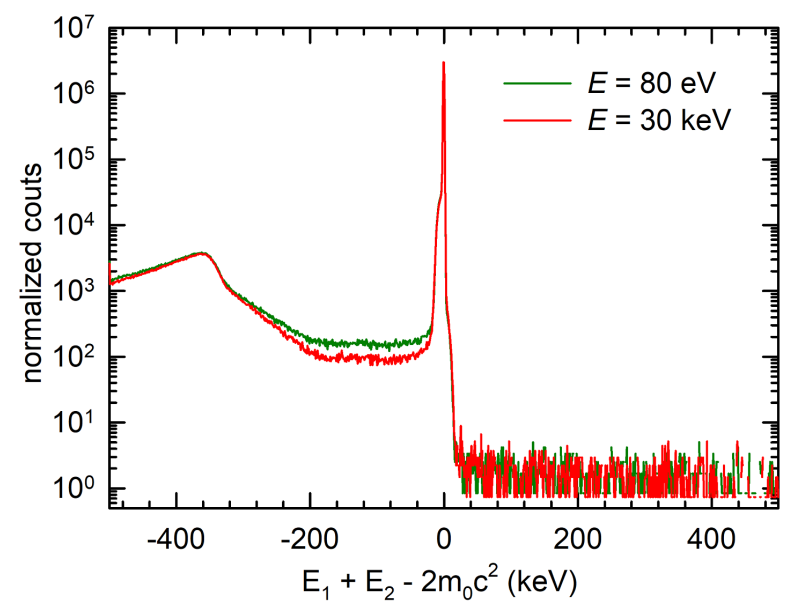

Fig. 8. Vertical cuts from normalized CDB spectra (in the range $\left.\left|E_{1}-E_{2}\right|<10 \mathrm{keV}\right)$ for the smooth Au film.
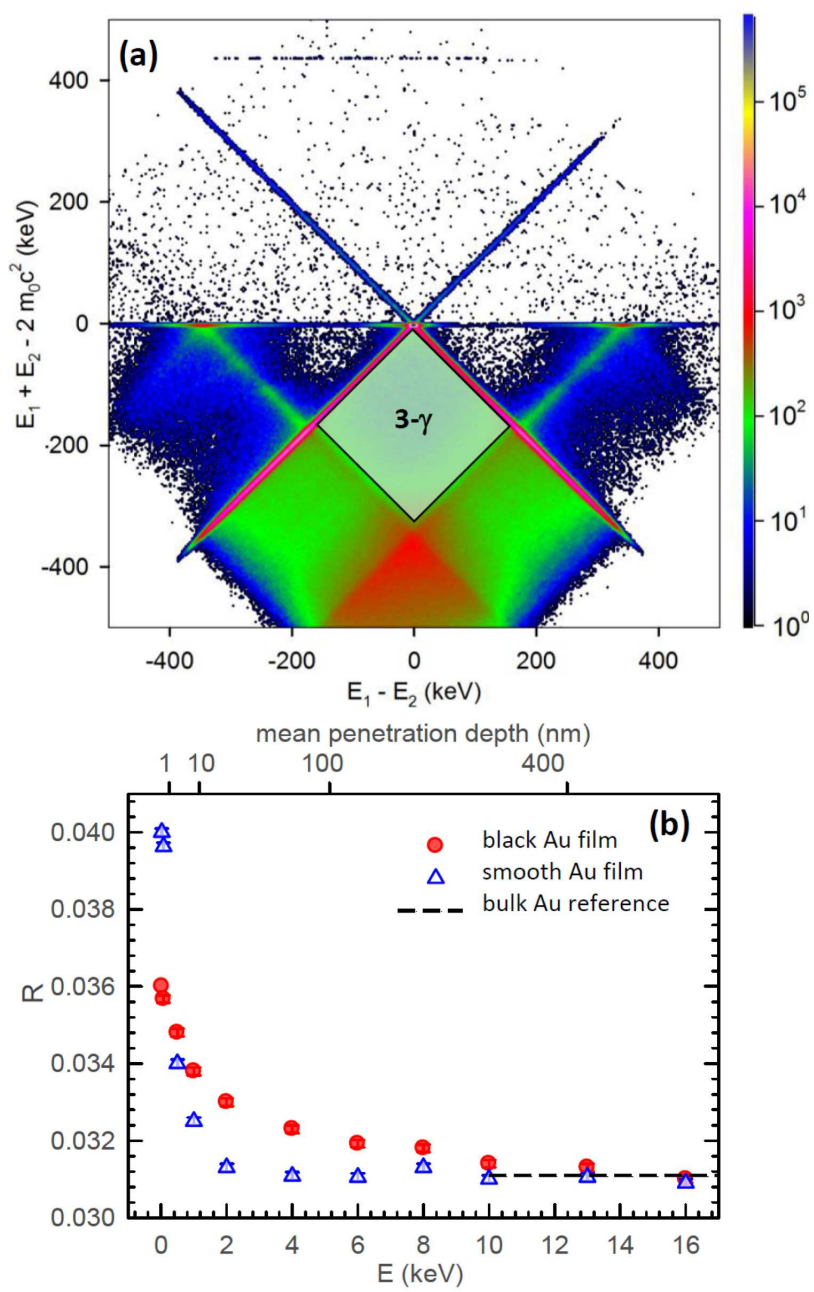

Fig. 9. (a) Schematic depiction of the region used for calculation of the area $A_{3-\gamma}$ in two-dimensional CDB spectrum, (b) the dependence of the $R$ parameter calculated from CDB spectra on the energy of incident positrons. Dashed line indicates the $R$ parameter value for the bulk Au reference.
Figure 8 shows vertical cuts in the range $\left|E_{1}-E_{2}\right|<$ $10 \mathrm{keV}$ from the CDB spectra for the smooth $\mathrm{Au}$ film normalized to the same total area. Enhanced contribution below the annihilation photo-peak is clearly visible in the spectrum measured with low energy of incident positrons, $E=80 \mathrm{eV}$. This is a contribution of $3-\gamma$ annihilations of $o$-Ps formed on surface.

Hence, the $R$ parameter providing a measure of $3-\gamma$ annihilation contribution can be calculated from twodimensional CDB spectra as the area in the region below the annihilation peak where $3-\gamma$ annihilation events contribute, see Fig. 9a, divided by the area of the annihilation photo-peak. The development of the $R$ parameter for smooth and black $\mathrm{Au}$ film calculated from CDB spectra is plotted in Fig. 9b. The $R(E)$ curves calculated from CDB spectra exhibit similar behavior to those obtained from single $\gamma$-ray spectra. The $3-\gamma$ contribution in the black $\mathrm{Au}$ is lower at the surface due to higher surface roughness and extends to significantly higher depths because of Ps formed in micro-cavities in the porous subsurface region. The $R$ parameter calculated from CDB spectra is considered to be more accurate since it is significantly less influenced by background.

\section{Conclusions}

Ps formation in conventional smooth $\mathrm{Au}$ film and porous black $\mathrm{Au}$ film was compared. In the smooth film Ps is formed on the surface only. The black Au film contains a sub-surface region with micro-cavities interconnected with surface and Ps is formed not only on the surface but also in the micro-cavities and travels through them towards the surface. The $3-\gamma$ annihilation of Ps leaves a clear signature in two-dimensional CDB spectra. The $R$ parameters representing a measure of $3-\gamma$ annihilation contribution were calculated from single $\gamma$-ray and two-dimensional CDB spectra and their behavior is similar. The $R$ parameters obtained from CDB spectra are more accurate since they are less influenced by background.

\section{Acknowledgments}

This work was supported by the Czech Science Agency (project P108/12/G043).

\section{References}

[1] A.H. Pfund Rev. Sci. Instrum. 1, 397 (1930).

[2] M. Novotný, P. Fitl, A. Krasilnikova Sytchkova, J. Bulî́r, J. Lančok, P. Pokorný Cent. Eur. J. Phys. 7, 327 (2009).

[3] T.V. Teperik, F.J. García de Abajo, A.G. Borisov, M. Abdelsalam, P.N. Bartlett, Y. Sugawara, J.J. Baumberg Nature Photon. 2, 299 (2008).

[4] D.W. Gidley, H.-G. Peng, R.S. Vallery Annu. Rev. Mater. Res. 36, 49 (2006).

[5] A. Seeger, F. Banhart Phys. Status Solidi A 102, 171 (1987).

[6] C.H. Hodges, M.J. Stott Phys. Rev. B7, 73 (1973). 
[7] K.G. Lynn, J.R. MacDonald, R.A. Boie, L.C. Feldman, J.D. Gabbe, M.F. Robbins, E. Bonderup, J. Golovchenko Phys. Rev. Lett. 38, 241 (1977).

[8] W. Anwand, G. Brauer, M. Butterling, H.R. Kissener, A. Wagner Def. Diff. Forum 331, 25 (2012).

[9] J. Č́ižek, M. Vlček, I. Procházka Nucl. Instrum. Methods Phys. Res. A 623, 982 (2010).

[10] Software for positron annihilation, Positron Annihilation Group, Charles University in Prague.

[11] P. Asoka-Kumar, M. Alatalo, V.J. Ghosh, A.C. Kruseman, B. Nielsen, K.G. Lynn Phys. Rev. Lett. 77, 2097 (1996).

[12] J. Č́́žek, M. Vlček, I. Procházka New J. Phys. 14, 035005 (2012).

[13] O. Melikhova, J. Čížek, P. Hruška, M. Vlček, I. Procházka, W. Anwand, M. Novotný, J. Bulír J. Phys. Conf. Series 791 , 0120185 (2017).

[14] A. van Veen, H. Schut, M. Clement, J.M.M. de Nijs, A. Kruseman, M.R. Ijpma Appl. Surf. Sci. 85, 216 (1995).
[15] P.J. Schulz, K.G. Lynn Rev. Mod. Phys. 60, 701 (1988).

[16] A. van Veen, R. Hakvoort, H. Schut, P. Mijnarends J. Phys. IV (France) 5, Cl-37 (1995).

[17] Y.C. Wu, J. Jiang, S.J. Wang, A. Kallis, P.G. Coleman Phys.Rev. B 84, 064123 (2011).

[18] A.P. Mills, L. Pfeiffer Phys. Rev. Lett. 43, 1961 (1979).

[19] D.W. Gidley, R. Mayer, W.E. Frieze, K.G. Lynn Phys. Rev. Lett. 58, 595 (1987).

[20] J. Č́ížek, M. Vlček, F. Lukáč, O. Melikhova, I. Procházka, W. Anwand, A. Wagner, M. Butterling, R. Krause-Rehberg J. Phys.: Conf. Series 505 012043 (2014).

[21] J. Dryzek, D. Singleton, T. Suzuki, R. Yu Am. J. Phys. 74, 49 (2006). 